\title{
Correction to: Relationship of living arrangement with the decline in functional capacity in elderly people by gender: a longitudinal observational study
}

Haruhiko Imamura ${ }^{1 *}$, Eiko Uchiyama ${ }^{2}$, Miki Akiyama ${ }^{3}$, Ikuyo Kaneko ${ }^{4}$, Toru Takebayashi ${ }^{5}$ and Yuji Nishiwaki ${ }^{1}$

\section{Correction to: Environ Health Prev Med (2020) 25: 15 https://doi.org/10.1186/s12199-020-00853-w}

Following publication of the original article [1], Table 1 was incorrectly published. Table 1 consists of three parts: Total, Men and Women; however, the first part "Total $(\mathrm{n}=744, \mathrm{n}=165, \mathrm{n}=749, \mathrm{n}=612, \mathrm{n}=357)$ " was captured incorrectly as part of the header. It should be moved to the top row of the table just like the Men and Women. The correct Table 1 is shown below.

The original article [1] has been updated.

\section{Author details}

'Department of Environmental and Occupational Health, School of Medicine,

Toho University, 5-21-16 Omori-Nishi, Ota-ku, Tokyo 143-8540, Japan.

${ }^{2}$ Graduate School of Media and Governance, Keio University, Kanagawa,

Japan. ${ }^{3}$ Faculty of Environment and Information Studies, Keio University, Kanagawa, Japan. ${ }^{4}$ Keio University, Kanagawa, Japan. ${ }^{5}$ Department of

Preventive Medicine and Public Health, School of Medicine, Keio University,

Tokyo, Japan.

Published online: 19 June 2020

\section{Reference}

1. Imamura H, Uchiyama E, Akiyama M, et al. Relationship of living

arrangement with the decline in functional capacity in elderly people by gender: a longitudinal observational study. Environ Health Prev Med. 2020 25:15. https://doi.org/10.1186/s12199-020-00853-w.

The original article can be found online at https://doi.org/10.1186/s12199020-00853-w.

* Correspondence: haruhiko.imamura@med.toho-u.ac.jp

'Department of Environmental and Occupational Health, School of Medicine, Toho University, 5-21-16 Omori-Nishi, Ota-ku, Tokyo 143-8540, Japan

Full list of author information is available at the end of the article

(c) The Author(s). 2020 Open Access This article is licensed under a Creative Commons Attribution 4.0 International License, which permits use, sharing, adaptation, distribution and reproduction in any medium or format, as long as you give appropriate credit to the original author(s) and the source, provide a link to the Creative Commons licence, and indicate if changes were made. The images or other third party material in this article are included in the article's Creative Commons licence, unless indicated otherwise in a credit line to the material. If material is not included in the article's Creative Commons licence and your intended use is not permitted by statutory regulation or exceeds the permitted use, you will need to obtain permission directly from the copyright holder. To view a copy of this licence, visit http://creativecommons.org/licenses/by/4.0/ The Creative Commons Public Domain Dedication waiver (http://creativecommons.org/publicdomain/zero/1.0/) applies to the data made available in this article, unless otherwise stated in a credit line to the data. 
Table 1 Characteristics of the study population at baseline

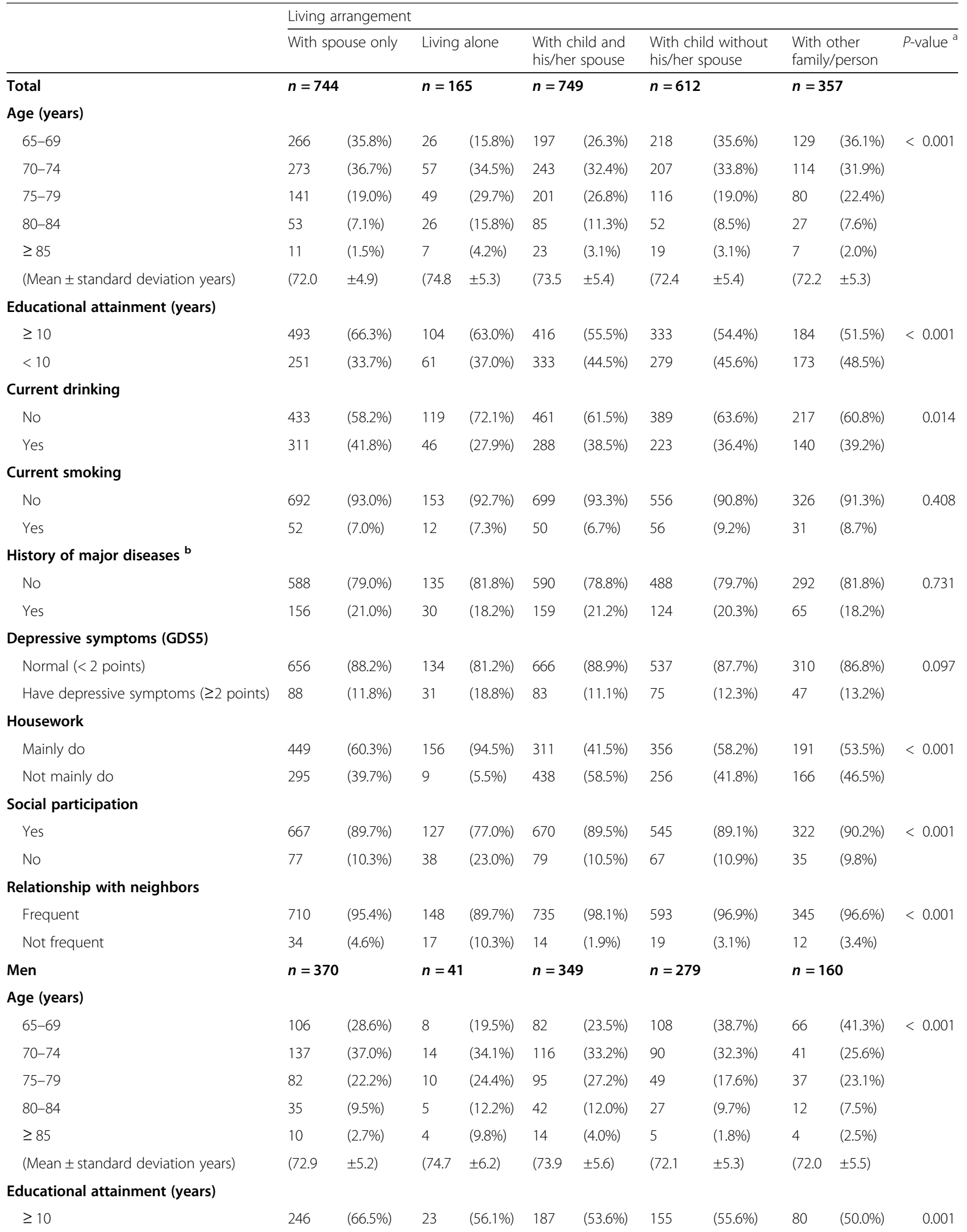


Table 1 Characteristics of the study population at baseline (Continued)

\begin{tabular}{|c|c|c|c|c|c|c|c|c|c|c|c|}
\hline \multirow[b]{3}{*}{$<10$} & \multicolumn{11}{|c|}{$\underline{\text { Living arrangement }}$} \\
\hline & \multicolumn{2}{|c|}{ With spouse only } & \multicolumn{2}{|c|}{ Living alone } & \multicolumn{2}{|c|}{$\begin{array}{l}\text { With child and } \\
\text { his/her spouse }\end{array}$} & \multicolumn{2}{|c|}{$\begin{array}{l}\text { With child without } \\
\text { his/her spouse }\end{array}$} & \multicolumn{2}{|c|}{$\begin{array}{l}\text { With other } \\
\text { family/person }\end{array}$} & \multirow[t]{2}{*}{$P$-value ${ }^{a}$} \\
\hline & 124 & (33.5\%) & 18 & $(43.9 \%)$ & 162 & $(46.4 \%)$ & 124 & $(44.4 \%)$ & 80 & $(50.0 \%)$ & \\
\hline \multicolumn{12}{|l|}{ Current drinking } \\
\hline No & 117 & (31.6\%) & 14 & (34.1\%) & 118 & (33.8\%) & 93 & (33.3\%) & 49 & $(30.6 \%)$ & \multirow[t]{2}{*}{0.940} \\
\hline Yes & 253 & $(68.4 \%)$ & 27 & (65.9\%) & 231 & $(66.2 \%)$ & 186 & $(66.7 \%)$ & 111 & $(69.4 \%)$ & \\
\hline \multicolumn{12}{|l|}{ Current smoking } \\
\hline No & 321 & $(86.8 \%)$ & 31 & (75.6\%) & 301 & (86.2\%) & 226 & $(81.0 \%)$ & 133 & (83.1\%) & \multirow[t]{2}{*}{0.111} \\
\hline Yes & 49 & $(13.2 \%)$ & 10 & $(24.4 \%)$ & 48 & $(13.8 \%)$ & 53 & $(19.0 \%)$ & 27 & $(16.9 \%)$ & \\
\hline \multicolumn{12}{|l|}{ History of major diseases ${ }^{b}$} \\
\hline No & 269 & $(72.7 \%)$ & 31 & (75.6\%) & 256 & $(73.4 \%)$ & 201 & $(72.0 \%)$ & 117 & (73.1\%) & \multirow[t]{2}{*}{0.990} \\
\hline Yes & 101 & (27.3\%) & 10 & $(24.4 \%)$ & 93 & $(26.6 \%)$ & 78 & $(28.0 \%)$ & 43 & $(26.9 \%)$ & \\
\hline \multicolumn{12}{|l|}{ Depressive symptoms (GDS5) } \\
\hline Normal (<2 points) & 332 & $(89.7 \%)$ & 30 & (73.2\%) & 308 & (88.3\%) & 253 & $(90.7 \%)$ & 136 & $(85.0 \%)$ & \multirow[t]{2}{*}{0.011} \\
\hline Have depressive symptoms ( $\geq 2$ points) & 38 & $(10.3 \%)$ & 11 & $(26.8 \%)$ & 41 & $(11.7 \%)$ & 26 & $(9.3 \%)$ & 24 & $(15.0 \%)$ & \\
\hline \multicolumn{12}{|l|}{ Housework } \\
\hline Mainly do & 84 & $(22.7 \%)$ & 35 & (85.4\%) & 59 & $(16.9 \%)$ & 69 & $(24.7 \%)$ & 42 & $(26.3 \%)$ & \multirow[t]{2}{*}{$<0.001$} \\
\hline Not mainly do & 286 & $(77.3 \%)$ & 6 & $(14.6 \%)$ & 290 & (83.1\%) & 210 & $(75.3 \%)$ & 118 & $(73.8 \%)$ & \\
\hline \multicolumn{12}{|l|}{ Social participation } \\
\hline Yes & 340 & (91.9\%) & 36 & (87.8\%) & 328 & (94.0\%) & 255 & (91.4\%) & 150 & $(93.8 \%)$ & \multirow[t]{2}{*}{0.485} \\
\hline No & 30 & $(8.1 \%)$ & 5 & $(12.2 \%)$ & 21 & $(6.0 \%)$ & 24 & $(8.6 \%)$ & 10 & $(6.3 \%)$ & \\
\hline \multicolumn{12}{|l|}{ Relationship with neighbors } \\
\hline Frequent & 351 & $(94.9 \%)$ & 36 & (87.8\%) & 342 & (98.0\%) & 265 & (95.0\%) & 151 & $(94.4 \%)$ & 0.020 \\
\hline Not frequent & 19 & $(5.1 \%)$ & 5 & $(12.2 \%)$ & 7 & $(2.0 \%)$ & 14 & $(5.0 \%)$ & 9 & $(5.6 \%)$ & \\
\hline Women & $n=3$ & & $n=12$ & & $n=4$ & & $n=3$ & & $n=1$ & & \\
\hline Age (years) & & & & & & & & & & & \\
\hline $65-69$ & 160 & $(42.8 \%)$ & 18 & $(14.5 \%)$ & 115 & $(28.8 \%)$ & 110 & $(33.0 \%)$ & 63 & $(32.0 \%)$ & $<0.001$ \\
\hline $70-74$ & 136 & $(36.4 \%)$ & 43 & (34.7\%) & 127 & (31.8\%) & 117 & (35.1\%) & 73 & $(37.1 \%)$ & \\
\hline $75-79$ & 59 & $(15.8 \%)$ & 39 & (31.5\%) & 106 & $(26.5 \%)$ & 67 & (20.1\%) & 43 & $(21.8 \%)$ & \\
\hline $80-84$ & 18 & $(4.8 \%)$ & 21 & $(16.9 \%)$ & 43 & $(10.8 \%)$ & 25 & $(7.5 \%)$ & 15 & $(7.6 \%)$ & \\
\hline$\geq 85$ & 1 & $(0.3 \%)$ & 3 & $(2.4 \%)$ & 9 & $(2.3 \%)$ & 14 & $(4.2 \%)$ & 3 & $(1.5 \%)$ & \\
\hline (Mean \pm standard deviation years) & $(71.0$ & $\pm 4.2)$ & $(74.9$ & $\pm 5.1)$ & $(73.2$ & $\pm 5.2)$ & $(72.8$ & $\pm 5.5)$ & $(72.4$ & $\pm 5.1)$ & \\
\hline Educational attainment (years) & & & & & & & & & & & \\
\hline$\geq 10$ & 247 & $(66.0 \%)$ & 81 & $(65.3 \%)$ & 229 & $(57.3 \%)$ & 178 & $(53.5 \%)$ & 104 & $(52.8 \%)$ & 0.002 \\
\hline$<10$ & 127 & $(34.0 \%)$ & 43 & (34.7\%) & 171 & $(42.8 \%)$ & 155 & $(46.5 \%)$ & 93 & $(47.2 \%)$ & \\
\hline Current drinking & & & & & & & & & & & \\
\hline No & 316 & $(84.5 \%)$ & 105 & (84.7\%) & 343 & $(85.8 \%)$ & 296 & $(88.9 \%)$ & 168 & $(85.3 \%)$ & 0.509 \\
\hline Yes & 58 & $(15.5 \%)$ & 19 & $(15.3 \%)$ & 57 & $(14.3 \%)$ & 37 & (11.1\%) & 29 & $(14.7 \%)$ & \\
\hline Current smoking & & & & & & & & & & & \\
\hline No & 371 & (99.2\%) & 122 & (98.4\%) & 398 & (99.5\%) & 330 & (99.1\%) & 193 & $(98.0 \%)$ & 0.428 \\
\hline Yes & 3 & $(0.8 \%)$ & 2 & $(1.6 \%)$ & 2 & $(0.5 \%)$ & 3 & $(0.9 \%)$ & 4 & $(2.0 \%)$ & \\
\hline History of major diseases ${ }^{b}$ & & & & & & & & & & & \\
\hline No & 319 & $(85.3 \%)$ & 104 & (83.9\%) & 334 & (83.5\%) & 287 & $(86.2 \%)$ & 175 & $(88.8 \%)$ & 0.491 \\
\hline Yes & 55 & $(14.7 \%)$ & 20 & $(16.1 \%)$ & 66 & $(16.5 \%)$ & 46 & $(13.8 \%)$ & 22 & $(11.2 \%)$ & \\
\hline
\end{tabular}


Table 1 Characteristics of the study population at baseline (Continued)

\begin{tabular}{|c|c|c|c|c|c|c|c|c|c|c|c|}
\hline \multirow{2}{*}{ Depressive symptoms (GDS5) } & \multicolumn{11}{|c|}{ Living arrangement } \\
\hline & \multicolumn{2}{|c|}{ With spouse only } & \multicolumn{2}{|c|}{ Living alone } & \multicolumn{2}{|c|}{$\begin{array}{l}\text { With child and } \\
\text { his/her spouse }\end{array}$} & \multicolumn{2}{|c|}{$\begin{array}{l}\text { With child without } \\
\text { his/her spouse }\end{array}$} & \multicolumn{2}{|c|}{$\begin{array}{l}\text { With other } \\
\text { family/person }\end{array}$} & $P$-value ${ }^{a}$ \\
\hline Normal (<2 points) & 324 & $(86.6 \%)$ & 104 & $(83.9 \%)$ & 358 & $(89.5 \%)$ & 284 & $(85.3 \%)$ & 174 & $(88.3 \%)$ & 0.340 \\
\hline Have depressive symptoms ( $\geq 2$ points) & 50 & $(13.4 \%)$ & 20 & $(16.1 \%)$ & 42 & $(10.5 \%)$ & 49 & $(14.7 \%)$ & 23 & $(11.7 \%)$ & \\
\hline \multicolumn{12}{|l|}{ Housework } \\
\hline Mainly do & 365 & $(97.6 \%)$ & 121 & $(97.6 \%)$ & 252 & $(63.0 \%)$ & 287 & $(86.2 \%)$ & 149 & (75.6\%) & $<0.001$ \\
\hline Not mainly do & 9 & $(2.4 \%)$ & 3 & $(2.4 \%)$ & 148 & $(37.0 \%)$ & 46 & $(13.8 \%)$ & 48 & $(24.4 \%)$ & \\
\hline \multicolumn{12}{|l|}{ Social participation } \\
\hline Yes & 327 & $(87.4 \%)$ & 91 & $(73.4 \%)$ & 342 & $(85.5 \%)$ & 290 & $(87.1 \%)$ & 172 & $(87.3 \%)$ & 0.002 \\
\hline No & 47 & $(12.6 \%)$ & 33 & $(26.6 \%)$ & 58 & $(14.5 \%)$ & 43 & $(12.9 \%)$ & 25 & $(12.7 \%)$ & \\
\hline \multicolumn{12}{|l|}{ Relationship with neighbors } \\
\hline Frequent & 359 & $(96.0 \%)$ & 112 & $(90.3 \%)$ & 393 & $(98.3 \%)$ & 328 & $(98.5 \%)$ & 194 & $(98.5 \%)$ & $<0.001$ \\
\hline Not frequent & 15 & $(4.0 \%)$ & 12 & $(9.7 \%)$ & 7 & $(1.8 \%)$ & 5 & $(1.5 \%)$ & 3 & $(1.5 \%)$ & \\
\hline
\end{tabular}

${ }^{a}$ Chi-square test

b History of major diseases was defined as having any one of the following diseases: stroke, myocardial infarction/angina, diabetes, Parkinson's disease, femoral neck fracture, and cancer 